\title{
Predictive value of eosinophil count on COVID-19 disease progression and outcomes, a retrospective study of Leishenshan Hospital in Wuhan, China.
}

\author{
Wei Xuan ${ }^{1}$, Diansan $\mathrm{Su}^{1}$, Xuliang Jiang ${ }^{1}$, Lili Huang ${ }^{1}$, Shuting Pan ${ }^{1}$, Caiyang Chen ${ }^{1}$, Xiao \\ Zhang ${ }^{1}$, Hui Zhu ${ }^{1}$, Song Zhang ${ }^{1}$, Weifeng $\mathrm{Yu}^{1}$, and Zhiyong Peng ${ }^{2}$ \\ ${ }^{1}$ Shanghai Jiao Tong University School of Medicine Affiliated Renji Hospital \\ ${ }^{2}$ Wuhan University Zhongnan Hospital
}

November 13, 2020

\begin{abstract}
Background: The potential protective role of eosinophils in the COVID-19 pandemic has aroused great interest, given their potential virus clearance function and the infection resistance of asthma patients to this coronavirus. However, it is unknown whether eosinophil counts could serve as a predictor of the severity of COVID-19. Methods: A total of 1004 patients with confirmed COVID-19 who were admitted to Leishenshan Hospital in Wuhan, China, were enrolled in this study, including 905 patients in the general ward and 99 patients in the ICU. We reviewed their medical data to analyze the association between eosinophils and intensive care unit (ICU) admission and death. Results: Of our 1004 patients with COVID-19, low eosinophil counts/ratios were observed in severe cases. After adjusting for confounders that could have affected the outcome, we found that eosinophil counts might not be a predictor of intensive care unit (ICU) admission. In 99 ICU patients, 58 of whom survived and 41 of whom died, low eosinophil level was an indicator of death in patients with severe COVID-19, with a cutoff value of $0.04 \times 109 / \mathrm{L}$ for the prediction of death. Conclusion: Our research revealed that a low eosinophil level was a predictor of death in ICU patients rather than a cause of ICU admission.
\end{abstract}

Predictive value of eosinophil count on COVID-19 disease progression and outcomes, a retrospective study of Leishenshan Hospital in Wuhan, China.

Running title: Eosinophil level was a predictor of death in ICU patients rather than a cause of ICU admission

Wei Xuan M.D., Ph.D. ${ }^{{ }^{*}}$, Xuliang Jiang, M.S. ${ }^{*}$, Lili Huang, M.S. ${ }^{1^{*}}$, Shuting Pan, Ph.D. ${ }^{2}$, Caiyang Chen M.D., Ph.D. ${ }^{1}$, Xiao Zhang, M.S. ${ }^{1}$, Hui Zhu M.D. ${ }^{1}$, Song Zhang M.D., Ph.D. ${ }^{1}$, Weifeng Yu M.D., Ph.D. ${ }^{1}$, Zhiyong Peng, M.D. ${ }^{3}$, and Diansan Su, M.D., Ph.D. ${ }^{1}$

${ }^{1}$ Department of Anesthesiology, Renji Hospital, Shanghai Jiaotong University, School of Medicine, Shanghai, China

${ }^{2}$ Clinical Center for Investigation, Renji Hospital, Shanghai Jiaotong University, School of Medicine, Shanghai, China

${ }^{3}$ Department of Critical Care Medicine, Zhongnan Hospital of Wuhan University, Wuhan, China

Wei Xuan, Xuliang Jiang and Lili Huang contributed equally to this work.

Corresponding Author: 
Diansan Su, Department of Anesthesiology, Renji Hospital, Shanghai Jiaotong University School of Medicine, 160 Pujian Road, Shanghai, 200127, China, diansansu@yahoo.com

Zhiyong Peng, Department of Critical Care Medicine, Zhongnan Hospital of Wuhan University, Wuhan, 430071, China, pengzy5@hotmail.com

\section{Abstract}

Background: The potential protective role of eosinophils in the COVID-19 pandemic has aroused great interest, given their potential virus clearance function and the infection resistance of asthma patients to this coronavirus. However, it is unknown whether eosinophil counts could serve as a predictor of the severity of COVID-19.

Methods: A total of 1004 patients with confirmed COVID-19 who were admitted to Leishenshan Hospital in Wuhan, China, were enrolled in this study, including 905 patients in the general ward and 99 patients in the ICU. We reviewed their medical data to analyze the association between eosinophils and intensive care unit (ICU) admission and death.

Results: Of our 1004 patients with COVID-19, low eosinophil counts/ratios were observed in severe cases. After adjusting for confounders that could have affected the outcome, we found that eosinophil counts might not be a predictor of intensive care unit (ICU) admission. In 99 ICU patients, 58 of whom survived and 41 of whom died, low eosinophil level was an indicator of death in patients with severe COVID-19, with a cutoff value of $0.04 \times 10^{9} / \mathrm{L}$ for the prediction of death.

Conclusion: Our research revealed that a low eosinophil level was a predictor of death in ICU patients rather than a cause of ICU admission.

Key words: COVID; eosinophil; asthma

Funding: This study was supported by grants from the National Natural Science Foundation of China (Nos. 81571030, 81771133,81970995, 81901985), Shanghai Pudong New Area Municipal Commission of Health and Family Planning Funding (PW2016D-4), Shanghai Jiao Tong University Integration Founding of Medicine and Engineering (YG2017MS53), Shanghai Shenkang Hospital Development Center Founding (SHDC12017X11), Renji Hospital Clinical Innovation Foundation (PYMDT-007), State Key Laboratoy of Neuroscience (SKLN-201803) and Renji Hospital founding (RJZZ18-019).

\section{Author statement:}

Wei Xuan : Conceptualization, Formal analysis, Project administration, Writing - original draft. Xuliang Jiang and Lili Huang: Formal analysis, Project administration, Writing - original draft. Shuting Pan : Data curation, Statistic analysis, Caiyang Chen : Formal analysis, Visualization. Software.Xiao Zhang : Data curation, Methodology. Hui Zhu : Data curation. Song Zhang : Data curation. Weifeng Yu : Supervision, Resources. Zhiyong Peng : Supervision, Resources.Diansan Su : Supervision, Conceptualization, Funding acquisition, Project administration.

Conflicts Of Interest: All authors declare no conflicts of interest.

\section{Abbreviations:}

WHO: World Health Organization

ICU: Intensive Care Unit

qRT-PCR: Quantitative reverse Rranscription-polymerase Thain Reaction

CDC: Disease Control and Prevention

IQR: Interquartile Range

ROC: Receiver Operating Characteristic 
OR: Odds Ratio

AUC: Area Under The Curve

CRP: Reactive Protein

APTT: Activated Partial Thromboplastin Time

WBC: White Blood Cell

PCT: Procalcitonin

ALB: Albumin

AST: Aspartate transaminase

\section{Introduction}

A newly identified coronavirus, COVID-19, has caused unexpected prevalence of respiratory disease for over half a year. The World Health Organization (WHO) pronounced COVID-19 a pandemic disease on March 11, 2020.[1] As of the day we completed this article, according to data from the WHO, there have been more than 30 million confirmed cases of COVID-19, causing 1 million deaths worldwide.

Given that $14 \%$ of patients have developed severe cases of the disease, efforts have been made to clarify the underlying infectious mechanisms and risk factors to reduce mortality.[2, 3] Owing to delayed virus clearance and a severe cytokine storm, severe acute respiratory distress syndrome and multiple organ failure accounted for the deterioration of most severe patients. [4] According to previous studies, [5-7] many factors have been proven to be predictive of severe cases, such as older age, male sex, hypertension, diabetes, excessive cytokines, coronary heart disease, and lymphocytopenia. However, many cases develop to severe status without these risk factors. Thus, identifying more risk factors is warranted.

Eosinophils are one of the less common blood leukocytes, [8] and they can be used to identify and predict the outcome of infectious diseases.[9] Eosinophils also play a central role in allergic disease,[10] in which levels are increased in the pathological processes of asthma and allergy.[11] Compared with other comorbidities, fewer patients with asthma have been found in the COVID-19 pandemic, which could be partly attributable to the virus-resistant function of eosinophils.[12] Although eosinophil levels might have vital clinical relevance to COVID-19 recovery because of their pro-inflammation and potential virus elimination properties, it is unknown whether eosinophil counts could serve as a predictor of the severity of COVID-19.[13] Given that a larger sample of clinical patients with COVID-19 was warranted,[14] we conducted a retrospective study involving 1004 patients with COVID-19 to analyze the association between eosinophils and intensive care unit (ICU) admission and death.

\section{Methods}

\section{Study design and patients}

We performed a single-center, retrospective review of patients admitted to Leishenshan Hospital in Wuhan, China, one of the hospitals designated to treat patients with COVID-19. A total of 1004 patients with confirmed COVID-19 were enrolled in this study, including 905 patients in the general ward and 99 patients in the ICU. All patients with COVID-19 pneumonia in this study were diagnosed according to the interim guidance for diagnosis and treatment provided by the National Health Commission of China and the WHO. All patients tested positive for COVID-19 by analyzing body fluid samples using quantitative reverse transcription-polymerase chain reaction (qRT-PCR). Patients' data were obtained by reviewing electronic medical records. This study was approved by the Ethics Commission of Renji Hospital (Ethical Committee approval number: KY2020-037). Informed consent was waived and approved by the Ethics Commission of Renji Hospital because of its retrospective nature.

\section{Data collection}


The medical records of all patients were independently obtained by the authors, who worked for the Critical Care Medicine Department of Leishenshan Hospital at that time, and laboratory data were reviewed from electronic medical records. All body fluid samples were analyzed and diagnosed by local health authorities as recommended by the Chinese Center for Disease Control and Prevention (CDC) and by using qRT-PCR with the CDC-approved process. The medical information collected included age, sex, laboratory values, and chronic disease histories (e.g., cardiac disease, cerebrovascular disease, pulmonary disease, malignancy, neurological disease, and diabetes).

\section{Statistical analysis}

For the baseline characteristics, categorical variables were summarized as frequencies and percentages, and continuous variables were described using median and interquartile range (IQR). Patients with ICU admission were matched with those without ICU admission at a 1:2 ratio based on their propensity scores, which were developed by considering variables that could potentially affect the outcome. The matching performance was assessed with the Kruskal-Wallis rank sum test, in which a $P$-value less than 0.05 was selected for adjustment in the following analysis.

To evaluate the difference in eosinophils between ICU and non-ICU patients, the optimal eosinophil cutoff point was determined based on receiver operating characteristic (ROC) curve analysis, and then, multivariate logistic regression was used, of which the results would be presented using an odds ratio (OR) with a $95 \%$ confidence interval for each covariate.

Prognostic analyses of ICU patients were conducted using logistic regression, as well as when in-hospital mortality was taken as the outcome variable, and eosinophils and other confounders were covariates. Covariates were selected by utilizing stepwise regression using the Akaike information criterion.

Statistical analyses were performed using $\mathrm{R}$ (version 4.0.0), and $P<.05$ was considered statistically significant.

\section{Results}

\section{Demographics and baseline characteristics of patients with COVID-19}

The baseline characteristics of all hospitalized patients are shown in Table 1. More male than female patients were found in the general ward and ICU: $21.6 \%$ more men than women $(P<.001)$. The patients' median age was 60 years (IQR, 49-69), with the median age of the patients in the general ward (58; IQR, 47-68) younger than that of those in the ICU $(69$; IQR, 62-80) $(P<.001)$. Among all patients, the most common comorbidities were hypertension $(27.8 \%)$, followed by diabetes $(11.8 \%)$, cardiovascular disease $(8.7 \%)$, pulmonary disease $(3.5 \%)$, stroke $(3.0 \%)$, chronic renal insufficiency $(2.7 \%)$, and chronic hepatitis and cirrhosis $(1.9 \%)$. Compared with the general ward, the comorbidities mentioned above were more commonly found in the ICU patients, whereas no significant difference was found in cancer rates between the two groups. In terms of laboratory results, higher counts and percentages of eosinophils and lymphocytes were shown in the general ward patients compared with those in the ICU. Lower white blood cell counts, neutrophil counts, and percentages were reported in the general ward patients $(P<.001)$ (Table 1$)$.

\section{Propensity score matching results showed that circulating eosinophil count was not an indicator for ICU admission}

In order to evaluate the role of eosinophil count in ICU admission after balancing the baseline characteristics of the patients in the general ward and ICU, we conducted propensity score matching of 70 non-ICU patients and 35 ICU patients. The matched baseline characteristics are described in Supplementary Table 1, in which the differences between groups are shown. Age, sex, and other comorbidities (e.g., coronary heart disease and diabetes) that could affect the outcome were balanced between the general ward and ICU patients. Other risk factors, including hypertension, C-reactive protein (CRP), urea, glucose, D-dimer, activated partial thromboplastin time (APTT), and procalcitonin, whose p-values were less than 0.05 were subsequently selected for adjustment. The predictive value of eosinophil counts was evaluated using ROC curves, and the optimal eosinophil cutoff point was $0.02 \times 10^{9} / \mathrm{L}$, which maximized the sum of sensitivity and specificity, 
leading to a 0.504 area under the curve (AUC) (Figure 1A). However, there was no significant difference between the effect of eosinophil count $<0.02 \times 10^{9} / \mathrm{L}$ and eosinophil count [?]0.02 $\times 10^{9} / \mathrm{L}$ on ICU admission (OR, 1.216; 95\% CI, 0.827-5.610) after considering other confounders. Only CRP played an important role in predicting the ICU admission of severe patients (OR, 1.013; 95\% CI, 1.001-1.026) (Table 2).

Demographics, baseline characteristics, and laboratory results of ICU patients with COVID-19

The baseline characteristics of the ICU patients on admission are shown in Table 3. There was no significant difference in sex between those who survived and those who died. The median age of the ICU patients was 69.0 years (IQR, 62.0-80.0), with the median age of those who died (73.0; IQR, 65.0-81.0) older than those who survived (66.0; IQR, 59.5-77.5) ( $P=.048)$. Most of the ICU patients were male $(68.7 \%)$, the male-to-female ratio was greater than $2: 1$, and most deaths (65.9\%) were of male patients. Among all ICU patients, nearly half (45.5\%) had hypertension, and some had other comorbidities, but there was no significant difference between the survival and death cases. The most frequent clinical symptoms were hypertension (45.5\%), cardiovascular disease $(21.2 \%)$, diabetes $(18.2 \%)$, stroke $(15.2 \%)$, chronic hepatitis and cirrhosis $(10.1 \%)$, pulmonary disease $(8.1 \%)$, chronic renal insufficiency $(8.1 \%)$, and malignancy $(2 \%)$.

With regard to the laboratory results, lower eosinophil counts and percentages were more commonly found in the COVID-19 deaths group compared with the survival group. In the death cases, some of the eosinophil counts even vanished, whereas this was rarely the case in the survival group. Higher white blood cell (WBC) counts, neutrophil counts and percentages, D-dimer, CRP, procalcitonin, and APTT were observed in the COVID-19 deaths group, while lower lymphocyte counts and percentages, and basophil and monocyte percentages were observed in this group. However, no significant differences were found in basophil and monocyte counts (Table 3 ).

\section{Circulating eosinophil count was a predictive factor for death in ICU patients with COVID-19}

The eosinophil predictive value was assessed using the ROC curve, which had an AUC of 0.665 and a cutoff value of $0.04 \times 10^{9}$ for distinguishing survival cases and death cases (Figure 1B). We divided the ICU patients into two groups depending on the circulating eosinophil counts: high eosinophil group ([?]0.04 x $\left.10^{9} / \mathrm{L}\right)$ and low eosinophil group $\left(<0.04 \times 10^{9} / \mathrm{L}\right)$. Multivariable logistic regression analysis showed that eosinophil counts, WBC counts, CRP, albumin, and APTT had a significant association with ICU patients' mortality, and specific results are expressed as ORs and $95 \%$ CIs in Table 4 . The results indicated that among the influencing factors, albumin had a protective effect, with an OR of 0.832. Remarkably, patients with lower eosinophil counts $\left(<0.04 \times 10^{9} / \mathrm{L}\right)$ were more likely to have fatal prognostic outcomes.

\section{Discussion}

Some 2011 patients were admitted to this hospital, more than 100 of these were admitted to the ICU during February 8 to April 15, 2020, and the patients involved in the current study were among these, for whom we obtained the medical records. Our results suggest that eosinophil counts can predict fatal outcomes for COVID-19 patients in the ICU, but contrary to what we hypothesized, eosinophil counts might not have the same predictive role for general ward patients with COVID-19.

Eosinophils are activated in parasitic infections, fungal infections, and viral infections. Previous research has shown that eosinopenia is an independent predictor of death in patients with pneumonia and has the capacity to protect against viral infection, $[15,16]$ but this protective effect only occurs in some circumstances. Circulating eosinophils normally range below 500 per microliter and could increase 20 -fold or more when they exert immune functions.[8] In patients with asthma, the accumulation of eosinophils in the lungs has risen 10 to 100 times compared with healthy volunteers,[17] whereas eosinopenia has appeared in patients with COVID-19.[18] On the basis of the existing data available worldwide, few asthmatic individuals have been vulnerable to COVID-19 infection, which has sparked special interest because asthma is characterized predominantly by eosinophilic inflammation.[19] This phenomenon could be attributable to the potential virus clearance ability of eosinophils and conventional therapeutics for asthma.[12] In accord with this observation, eosinopenia was more prominent in patients with severe COVID-19 infection.[18, 20, 21] Moreover, 
blood eosinophil counts have correlated with lymphocytes in all patients,[22] and normalization of eosinophil numbers followed the improvement of clinical status.[23] The studies mentioned above have revealed the prognostic indication function of eosinophil levels in patients with COVID-19, but whether eosinophil accumulation in the respiratory system or overall elevation in the human body increases COVID-19 virus resistance has not yet been clarified. An urgent question is also whether the eosinophil level could alter the course of COVID-19 or whether it has only an accompanying role during the infection process.[13] A recent retrospective study on eosinophils reviewed patients who visited the fever clinics of Shanghai General Hospital from late January to early February, 2020.[24] This study revealed that the eosinophil count in 12 confirmed patients with COVID-19 from 227 fever clinic outpatients was lower than that in those with other types of pneumonia. In hospitalized patients, eosinophil counts fell below the detection limit in all 12 severe patients, and low eosinophil counts could be related to severe conditions.

The present retrospective study involved 1004 patients within our authority to track their medical records. We found that the primary characteristics of ICU patients included male sex, old age, hypertension, stroke, high WBCs, low neutrophil and lymphocyte counts/ratios, and low eosinophil counts/ratios. These findings are comparable to prior publications.[5-7] To explore whether eosinophil levels could alter the clinical course of patients with mild COVID-19 infection, we conducted a matching study of 35 ICU and 70 general ward patients, all of whom were first admitted to the general ward of Leishenshan Hospital. Thus, we could track records of their earliest eosinophil level. In a matching study, we balanced several variables that could affect the outcome to reduce interference. After balancing, the risk factors that potentially promote infection from mild to severe status were hypertension, CRP, urea, D-dimer, APTT, procalcitonin, and glucose. It is noteworthy that there was no significant difference between the effect of eosinophil counts $<0.02 \times 10^{9} / \mathrm{L}$ and eosinophil counts [?]0.02 $\times 10^{9} / \mathrm{L}$ on ICU admission after taking other confounders into consideration.

To further define the role of eosinophils in patients with severe COVID-19, we divided 99 ICU patients into 58 survival and 41 death cases. Not surprisingly, old age was the vital risk factor for death compared with other comorbidities. With regard to laboratory data, low eosinophil levels, along with several other markers, such as CRP, were predictors of fatal outcomes in patients with severe COVID-19. In the present study, the eosinophil counts had an AUC of 0.665 , and the cutoff value was 0.04 for the prediction of death in ICU patients with COVID-19, whereas others have reported eosinophil counts with an AUC of 0.74 and a cutoff value of 0.015 for the diagnosis of COVID-19 among 109 confirmed cases and 215 other types of pneumonia.[24] The death OR of eosinophil counts below $0.04 \times 10^{9} / \mathrm{L}$ was 4.087 after multivariable logistic regression analysis in our study.

Our results suggest a predictive value of eosinophils for fatal outcomes in ICU patients. However, a recent study from Columbia University that included 1298 patients with COVID-19 with an asthma prevalence of $12.6 \%$ failed to show a significant difference in length of hospital stay, need for intubation, and mortality between asthma and non-asthma patients.[25] This observation challenges the basic assumption of the protective effect of eosinophils in patients with asthma. Therefore, further prospective studies are urgently required.

\section{Limitations}

The limitation of this study is that owing to its retrospective nature, we could not review the basic eosinophil count for all ICU patients, given that some of them were transferred to the ICU directly from other hospitals or their medical records were not tracked in the general wards.

\section{Conclusions}

Our findings suggest that eosinophil counts might not be predictive of ICU admission but could indicate a death outcome for ICU patients. There was no significant difference in eosinophil counts higher or lower than $0.02 \times 10^{9} / \mathrm{L}$ on ICU admission among general ward patients, whereas eosinophil counts below $0.04 \mathrm{x}$ $10^{9} / \mathrm{L}$ were more likely to have fatal outcomes in ICU patients. Prospective research and more patients are needed to further explore the exact role of eosinophils in COVID-19. 


\section{References:}

1. Vabret N, Britton GJ, Gruber C, et al. Immunology of COVID-19: Current State of the Science. Immunity. 2020;52(6):910-941.

2. Zochios V, Brodie D, Charlesworth M, Parhar KK. Delivering extracorporeal membrane oxygenation for patients with COVID-19: what, who, when and how? Anaesthesia. 2020;75(8):997-1001.

3. Wu A, Peng Y, Huang B, et al. Genome Composition and Divergence of the Novel Coronavirus (2019nCoV) Originating in China. Cell Host Microbe. 2020;27(3):325-328.

4. Ye Q, Wang B, Mao J. Cytokine Storm in COVID-19 and Treatment.Journal of Infection. 2020;80(6).

5. Liu J, Li S, Liu J, et al. Longitudinal characteristics of lymphocyte responses and cytokine profiles in the peripheral blood of SARS-CoV-2 infected patients. EBioMedicine. 2020;55:102763.

6. Wan S, Yi Q, Fan S, et al. Relationships among lymphocyte subsets, cytokines, and the pulmonary inflammation index in coronavirus (COVID-19) infected patients. Br J Haematol. 2020;189(3):428-437.

7. Steffens I. A hundred days into the coronavirus disease (COVID-19) pandemic. Euro Surveill. 2020;25(14).

8. Klion AD, Ackerman SJ, Bochner BS. Contributions of Eosinophils to Human Health and Disease. Annu Rev Pathol. 2020;15:179-209.

9. Karakonstantis S, Gryllou N, Papazoglou G, Lydakis C. Eosinophil count (EC) as a diagnostic and prognostic marker for infection in the internal medicine department setting. Rom J Intern Med.2019;57(2):166174.

10. Eng SS, DeFelice ML. The Role and Immunobiology of Eosinophils in the Respiratory System: a Comprehensive Review. Clin Rev Allergy Immunol. 2016;50(2):140-158.

11. Wardlaw AJ, Brightling C, Green R, Woltmann G, Pavord I. Eosinophils in asthma and other allergic diseases. Br Med Bull.2000;56(4):985-1003.

12. Liu S, Zhi Y, Ying S. COVID-19 and Asthma: Reflection During the Pandemic. Clin Rev Allergy Immunol. 2020;59(1):78-88.

13. Lindsley AW, Schwartz JT, Rothenberg ME. Eosinophil responses during COVID-19 infections and coronavirus vaccination. J Allergy Clin Immunol. 2020;146(1):1-7.

14. Qian GQ, Zhang X, Ma AHY, Yang NB. Response to: Eosinophil count in severe coronavirus disease 2019. Qjm. 2020;113(7):513-514.

15. Rosenberg HF, Dyer KD, Domachowske JB. Eosinophils and their interactions with respiratory virus pathogens. Immunol Res.2009;43(1-3):128-137.

16. Echevarria C, Hartley T, Nagarajan T, et al. 30 day mortality and eosinopenia in patients with pneumonia. European Respiratory Journal. 2014.

17. Farahi N, Loutsios C, Tregay N, et al. In vivo imaging reveals increased eosinophil uptake in the lungs of obese asthmatic patients.J Allergy Clin Immunol. 2018;142(5):1659-1662.e1658.

18. Qin C, Zhou L, Hu Z, et al. Dysregulation of Immune Response in Patients With Coronavirus 2019 (COVID-19) in Wuhan, China. Clin Infect Dis. 2020;71(15):762-768.

19. Postma DS, Rabe KF. The Asthma-COPD Overlap Syndrome. N Engl J Med. 2015;373(13):1241-1249.

20. Liu F, Xu A, Zhang Y, et al. Patients of COVID-19 may benefit from sustained Lopinavir-combined regimen and the increase of Eosinophil may predict the outcome of COVID-19 progression. Int $J$ Infect Dis.2020;95:183-191. 
21. Du Y, Tu L, Zhu P, et al. Clinical Features of 85 Fatal Cases of COVID-19 from Wuhan. A Retrospective Observational Study. Am J Respir Crit Care Med. 2020;201(11):1372-1379.

22. Zhang JJ, Dong X, Cao YY, et al. Clinical characteristics of 140 patients infected with SARS-CoV-2 in Wuhan, China. Allergy.2020;75(7):1730-1741.

23. Jesenak M, Banovcin P, Diamant Z. COVID-19, chronic inflammatory respiratory diseases and eosinophils-Observations from reported clinical case series. Allergy. 2020;75(7):1819-1822.

24. Xie G, Ding F, Han L, Yin D, Lu H, Zhang M. The role of peripheral blood eosinophil counts in COVID-19 patients. Allergy. 2020.

25. Lovinsky-Desir S, Deshpande DR, De A, et al. Asthma among hospitalized patients with COVID-19 and related outcomes. J Allergy Clin Immunol. 2020.

Table 1. Demographics, baseline characteristics and laboratory results of all patients with COVID-19

\begin{tabular}{|c|c|c|c|c|}
\hline Characteristics & $\begin{array}{l}\text { All patients } \\
(\mathrm{n}=1004)\end{array}$ & $\begin{array}{l}\text { General ward } \\
(\mathrm{n}=905)\end{array}$ & ICU $(n=99)$ & $p$ value \\
\hline Male, n (\%) & $494(49.2 \%)$ & $426(47.1 \%)$ & $68(68.7 \%)$ & $<0.001$ \\
\hline $\begin{array}{l}\text { Age, median } \\
\text { (range) }\end{array}$ & $60(49-69)$ & $58(47-68)$ & $69(62-80)$ & $<0.001$ \\
\hline \multicolumn{5}{|l|}{$\begin{array}{l}\text { Any } \\
\text { comorbidity, } \mathbf{n} \\
(\%)\end{array}$} \\
\hline $\begin{array}{l}\text { Hypertension, n } \\
(\%)\end{array}$ & $280(27.8 \%)$ & $235(25.9 \%)$ & $45(45.5 \%)$ & $<0.001$ \\
\hline Diabetes, n (\%) & $119(11.8 \%)$ & $101(11.1 \%)$ & $18(18.2 \%)$ & 0.04 \\
\hline $\begin{array}{l}\text { Cardiovascular } \\
\text { disease, n (\%) }\end{array}$ & $88(8.7 \%)$ & $67(7.4 \%)$ & $21(21.2 \%)$ & $<0.001$ \\
\hline $\begin{array}{l}\text { Pulmonary } \\
\text { disease, n (\%) }\end{array}$ & $35(3.5 \%)$ & $27(3.0 \%)$ & $8(8.1 \%)$ & 0.019 \\
\hline Stroke, n (\%) & $30(3.0 \%)$ & $15(1.7 \%)$ & $15(15.2 \%)$ & $<0.001$ \\
\hline $\begin{array}{l}\text { Malignancy, n } \\
(\%)\end{array}$ & $13(1.3 \%)$ & $11(1.2 \%)$ & $2(2 \%)$ & 0.838 \\
\hline $\begin{array}{l}\text { Chronic renal } \\
\text { insufficiency, n } \\
(\%)\end{array}$ & $27(2.7 \%)$ & $19(2.1 \%)$ & $8(8.1 \%)$ & 0.002 \\
\hline $\begin{array}{l}\text { Chronic hepatitis } \\
\text { and cirrhosis, } \mathrm{n} \\
(\%)\end{array}$ & $36(1.9 \%)$ & $26(2.9 \%)$ & $10(10.1 \%)$ & 0.001 \\
\hline $\begin{array}{l}\text { White blood cell } \\
\text { count } \\
\left(3.5-9.5 \times 10^{9} / \mathrm{L}\right)\end{array}$ & $5.68(4.7-7.09)$ & $5.56(4.63-6.78)$ & $9.53(6.15-11.82)$ & $<0.001$ \\
\hline $\begin{array}{l}\text { Neutrophil count } \\
\left(1.8-6.3 \times 10^{9} / \mathrm{L}\right)\end{array}$ & $3.31(2.55-4.48)$ & $3.16(2.51-4.12)$ & $7.48(4.48-10.48)$ & $<0.001$ \\
\hline $\begin{array}{l}\text { Neutrophil } \\
\text { percentage } \\
(40-75 \%)\end{array}$ & $58.9(52.35-67.35)$ & $57.95(51.6-63.92)$ & $84.3(73.5-89.7)$ & $<0.001$ \\
\hline $\begin{array}{l}\text { Lymphocyte } \\
\text { count } \\
\left(1.1-3.2 \times 10^{9} / \mathrm{L}\right)\end{array}$ & $1.56(1.16-1.94)$ & $1.61(1.26-1.99)$ & $0.79(0.5-1.2)$ & $<0.001$ \\
\hline
\end{tabular}




$\begin{array}{lcccc}\begin{array}{l}\text { Lymphocyte } \\ \text { percentage } \\ (20-50 \%)\end{array} & 28.1(21.1-34.1) & 29.3(23.7-34.9) & 8.4(5.5-15.6) & <0.001 \\ \begin{array}{l}\text { Eosinophil count } \\ (0.02-\end{array} & 0.1(0.06-0.17) & 0.11(0.06-0.18) & 0.03(0.0-0.13) & <0.001 \\ \begin{array}{l}\left.0.52 \times 10^{9} / \mathrm{L}\right) \\ \begin{array}{l}\text { Eosinophil } \\ \text { percentage }\end{array}\end{array} & 1.8(1-3) & 1.9(1.1-3.12) & 0.2(0-1.5) & <0.001 \\ (0.4-8 \%) & & & \end{array}$

Data are the median (IQR) or $n / N(\%)$. $P$ values comparing general ward cases and ICU cases are from $\chi 2$, Fisher's exact test, or Mann-Whitney U test. The frequencies of categorical variables were compared using the chi-square and Fisher's exact test as appropriate.

Table 2. Multivariable logistic regression ORs (95\%CI) for ICU admission

\begin{tabular}{llll}
\hline Covariates & Odds Ratio & $\mathbf{9 5 \%}$ CI & p value \\
Hypertension & 2.149 & $(0.827,5.61)$ & 0.114 \\
CRP & 1.013 & $(1.001,1.026)$ & 0.039 \\
Urea & 0.997 & $(0.973,1.02)$ & 0.816 \\
GLU & 1.033 & $(0.946,1.143)$ & 0.496 \\
D_dimer & 1.01 & $(0.978,1.041)$ & 0.491 \\
APTT & 1.001 & $(0.983,1.016)$ & 0.895 \\
PCT & 1.02 & $(0.716,1.457)$ & 0.906 \\
EOS count & & & 0.732 \\
$\geq 0.02 \times 10^{9} / \mathrm{L}$ & 1 & & \\
$<0.02 \times 10^{9} / \mathrm{L}$ & 1.216 & $(0.38,3.659)$ & \\
\hline
\end{tabular}

CRP: C-reactive protein; GLU: Glucose; APTT: Activated partial thromboplastin time; PCT: Procalcitonin.

Table 3. Demographics, baseline characteristics and laboratory results of patients with COVID-19 in ICU

\begin{tabular}{|c|c|c|c|c|}
\hline Characteristics & $\begin{array}{l}\text { All ICU patients } \\
(\mathrm{n}=99)\end{array}$ & $\begin{array}{l}\text { Survival cases } \\
(n=58)\end{array}$ & $\begin{array}{l}\text { Death cases } \\
(\mathrm{n}=41)\end{array}$ & $\mathrm{p}$ value \\
\hline Male, n (\%) & $68(68.7 \%)$ & $41(70.7 \%)$ & $27(65.9 \%)$ & 0.609 \\
\hline $\begin{array}{l}\text { Age,median } \\
\text { (range) }\end{array}$ & $69(62-80)$ & $66(59.5-77.5)$ & $73(65-81)$ & 0.048 \\
\hline \multicolumn{5}{|l|}{$\begin{array}{l}\text { Any } \\
\text { comorbidity, n } \\
\text { (\%) }\end{array}$} \\
\hline $\begin{array}{l}\text { Hypertension, } \mathrm{n} \\
(\%)\end{array}$ & $45(45.5 \%)$ & $23(39.7 \%)$ & $22(53.7 \%)$ & 0.168 \\
\hline Diabetes, n (\%) & $18(18.2 \%)$ & $11(19 \%)$ & $7(17.1 \%)$ & 0.810 \\
\hline $\begin{array}{l}\text { Cardiovascular } \\
\text { disease, n (\%) }\end{array}$ & $21(21.2 \%)$ & $11(19 \%)$ & $10(24.4 \%)$ & 0.515 \\
\hline $\begin{array}{l}\text { Pulmonary } \\
\text { disease, } \mathrm{n}(\%)\end{array}$ & $8(8.1 \%)$ & $3(5.2 \%)$ & $5(12.2 \%)$ & 0.374 \\
\hline Stroke, n (\%) & $15(15.2 \%)$ & $10(17.2 \%)$ & $5(12.2 \%)$ & 0.490 \\
\hline $\begin{array}{l}\text { Malignancy, n } \\
\text { (\%) }\end{array}$ & $2(2 \%)$ & $1(1.7 \%)$ & $1(2.4 \%)$ & 1.000 \\
\hline
\end{tabular}




\begin{tabular}{|c|c|c|c|c|}
\hline $\begin{array}{l}\text { Chronic renal } \\
\text { insufficiency, n } \\
(\%) \\
\text { Laboratory } \\
\text { results }\end{array}$ & $8(8.1 \%)$ & $3(5.2 \%)$ & $5(12.2 \%)$ & 0.374 \\
\hline $\begin{array}{l}\text { White blood cell } \\
\text { count }\left(\times 10^{9} / \mathrm{L}\right)\end{array}$ & $9.53(6.15-11.82)$ & $7.11(5.58-9.82)$ & $11.41(9.66-15.59)$ & ¡0.001 \\
\hline $\begin{array}{l}\text { Neutrophil count } \\
\left(\times 10^{9} / \mathrm{L}\right)\end{array}$ & $7.48(4.48-10.48)$ & $5.42(4.22-8.23)$ & $10.12(8.36-13.74)$ & ¡0.001 \\
\hline $\begin{array}{l}\text { Neutrophil } \\
\text { percentage (\%) }\end{array}$ & $84.3(73.5-89.7)$ & $\begin{array}{l}78.85 \\
(68.98-84.38)\end{array}$ & $88.7(86.3-92.9)$ & ¡0.001 \\
\hline $\begin{array}{l}\text { Lymphocyte } \\
\text { count }\left(\times 10^{9} / \mathrm{L}\right)\end{array}$ & $0.79(0.5-1.2)$ & $0.96(0.59-1.32)$ & $0.6(0.40-0.96)$ & 0.004 \\
\hline $\begin{array}{l}\text { Lymphocyte } \\
\text { percentage }(\%)\end{array}$ & $8.4(5.5-15.6)$ & $12.45(8.08-18.28)$ & $5.5(3.8-8.25)$ & ¡0.001 \\
\hline $\begin{array}{l}\text { Eosnophil count } \\
\left(\times 10^{9} / \mathrm{L}\right)\end{array}$ & $0.03(0.0-0.13)$ & $0.08(0.01-0.15)$ & $0.01(0.0-0.07)$ & 0.005 \\
\hline $\begin{array}{l}\text { Eosnophil } \\
\text { percentage (\%) }\end{array}$ & $0.2(0-1.5)$ & $1.05(0.18-2.08)$ & $0.03(0-0.35)$ & ¡0.001 \\
\hline $\begin{array}{l}\text { Basophil count } \\
\left(\times 10^{9} / \mathrm{L}\right)\end{array}$ & $0.02(0.01-0.04)$ & $0.02(0.01-0.04)$ & $0.02(0.01-0.04)$ & 0.844 \\
\hline $\begin{array}{l}\text { Basophil } \\
\text { percentage (\%) }\end{array}$ & $0.2(0.1-0.4)$ & $0.25(0.2-0.5)$ & $0.2(0.1-0.3)$ & 0.016 \\
\hline $\begin{array}{l}\text { Monocyte count } \\
\left(\times 10^{9} / \mathrm{L}\right)\end{array}$ & $0.51(0.37-0.72)$ & $0.52(0.40-0.72)$ & $0.51(0.3-0.72)$ & 0.706 \\
\hline $\begin{array}{l}\text { Monocyte } \\
\text { percentage (\%) }\end{array}$ & $6.1(4.0-8.0)$ & $7.3(5.65-8.85)$ & $4.6(3.15-6.15)$ & ¡0.001 \\
\hline D-dimer (mg/L) & $3.46(1.74-7.24)$ & $2.89(1.28-4.86)$ & $6.05(3.18-13.11)$ & ¡0.001 \\
\hline CRP (mg/L) & $35.35(21.67-57.7)$ & $29.74(7.86-35.85)$ & $42.2(35.35-112.6)$ & ¡0.001 \\
\hline PCT (ng/ml) & $0.28(0.1-0.63)$ & $0.15(0.07-0.36)$ & $0.60(0.28-1.49)$ & ¡0.001 \\
\hline APTT (seconds) & $33.6(30.2-41.3)$ & $33.4(30.45-38.5)$ & $38.2(29.85-47.15)$ & 0.017 \\
\hline
\end{tabular}

Data are the median (IQR) or $n / N(\%)$. $P$ values comparing severe cases and moderate cases are from $\chi 2$, Fisher's exact test, or Mann-Whitney U test. The frequencies of categorical variables were compared using the chi-square and Fisher's exact test as appropriate. CRP: C-reactive protein; PCT: Procalcitonin; APTT: Activated partial thromboplastin time.

Table 4. Multivariable logistic regression ORs $(95 \% \mathrm{CI})$ for death risk factors of ICU patients

\begin{tabular}{llll}
\hline Covariates & Odds Ratio & $\mathbf{9 5 \%}$ CI & p value \\
Eos count $\left(0.04 \times 10^{9} / \mathrm{L}\right)$ & 4.087 & $(1.089,17.839)$ & 0.045 \\
WBC count & 1.266 & $(1.095,1.521)$ & 0.005 \\
CRP & 1.035 & $(1.013,1.063)$ & 0.005 \\
ALB & 0.832 & $(0.72,0.943)$ & 0.006 \\
APTT & 1.106 & $(1.034,1.194)$ & 0.006 \\
\hline
\end{tabular}

WBC: White blood cell; CRP: C-reactive protein; ALB: albumin; APTT: Activated partial thromboplastin time.

Fig 1. ROC curve analysis of predictive value of EOS for ICU admission and death of ICU patients. 
(A) EOS counts had AUC of 0.504 and the cutoff value was $0.02 \times 10^{9} / \mathrm{L}$ for prediction of ICU admission, the sensitivity was 0.800 and specificity was 0.3143 ; (B) EOS counts had AUC of 0.665 and the cutoff value was $0.04 \times 10^{9} / \mathrm{L}$ for prediction of death of ICU patients, the sensitivity was 0.569 and specificity was 0.7317 .
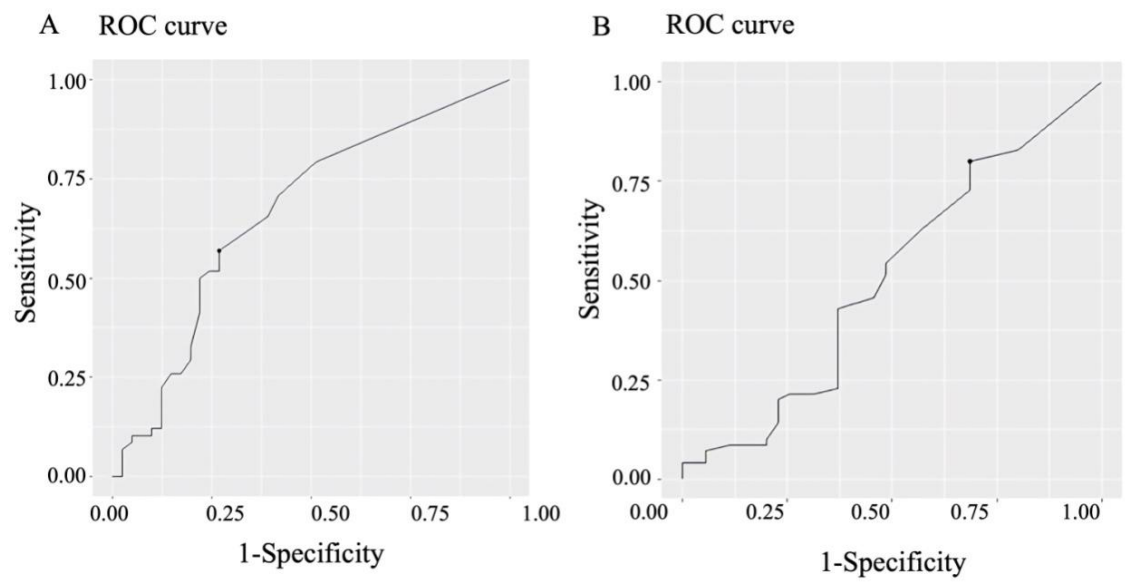